\title{
On the Complex Formation Between Thorium and Acetylacetone
}

\author{
JAN R Y D B E R G \\ FOA 1, Ulriksdal, and Institute of Inorganic and Physical Chemistry, \\ University of Stockholm. Sweden
}

$I^{t}$

was once believed that complex compounds are formed directly in one step like $\mathrm{Me}+m \mathrm{X} \rightarrow \mathrm{MeX}_{m}$, where $\mathrm{Me}$ is a metal ion and $\mathrm{X}$ is an uncharged molecule or a negative ion (ligand). Accordingly no other complexes would be been formed in appreciable amounts.

During the last few decades a number of complex systems have been thoroughly studied; in all cases not only the complex with maximum number $m$ of ligands appears to be formed, but also, to some extent, all intermediate steps $\mathrm{MeX}_{n}$, where $n=1,2, \ldots, m-1$. This is true both for systems with inorganic ions like $\mathrm{Cr}^{3+}-\mathrm{SCN}^{-(1)}$ and $\mathrm{Hg}^{2+}-\mathrm{Cl}^{-(2)}$, for systems where the ligand is uncharged, like metal-ammine systems ${ }^{(3)}$, and for systems involving organic ions like $\mathrm{Cu}^{2+}-\mathrm{CH}_{3} \mathrm{COO}^{-(4)}$.

Organic molecules with two coordinating groups (e.g. salicylaldehyde, oxine, cupferron, acetylacetone) combine with metals to form so-called chelates, or inner complexes. One might suspect that these chelates will be formed in the same stepwise manner. Recently Calvin and Wilson ${ }^{5}$ have calculated equilibrium constants for the formation of a great number of copper(II) chelates, $\mathrm{CuX}_{2}$ and the intermediate step $\mathrm{CuX}^{-}$, in a mixture of dioxane and water; in most cases the accuracy was rather low. However there is still a widespread opinion that in the formation of chelates only the uncharged complex is obtained. Hückel ${ }^{6}$, when discussing the formation of acetylacetone chelates, states that "Die Zahl der innerkomplex gebundenen Acetylacetonreste entspricht fast durchweg der Wertigkeit der als Zentralatom fungierenden Metalles, so dass sich die allgemeine Formel für die Acetylacetonate $\mathrm{Me}\left(\mathrm{CH}_{3} \mathrm{COCH}: \mathrm{COCH}_{3}\right)_{m}$ in der $m$ die Wertigkeit des Metalles bedeutet, aufstellen lässt." 
In this paper the results are given of an investigation of the complex formation between $\mathrm{Th}^{4+}$ and acetylacetone (HAa). The data could be explained well only by assuming that all complexes $\mathrm{ThAa}^{3+}, \mathrm{ThAa}_{2}^{2+}, \mathrm{ThAa}_{3}^{+}$and $\mathrm{ThAa}_{4}$ are formed; with this assumption the mass action constants for the reaction steps could be calculated.

\section{METHOD OF INVESTIGATION}

To be able to calculate the constants for a complex equilibrium one has to know the total amount of central atom and ligand and the free concentration of at least one definite species. Sometimes emf measurements have been used to find the concentration of central ion or ligand. This was not possible in the present case, as no reproducible thorium or acetylacetonate electrode had been constructed. Nor could optical methods be used, at least not with visible light, since the thorium-acetylacetone complexes are uncoloured.

In his classical investigation on the complex formation between $\mathrm{Hg}^{2+}$ and $\mathrm{X}^{-}\left(\mathrm{X}=\mathrm{Cl}^{-}, \mathrm{Br}^{-}, \mathrm{I}^{-}\right)$Morse ${ }^{7}$ made use of the fact that $\mathrm{HgX}_{2}$ molecules, but not ions, are soluble in toluene. From the distribution of mercury between toluene and water as a function of $\left[\mathrm{X}^{-}\right]$he could calculate the concentration of $\mathrm{HgX}^{+}$and $\mathrm{HgX}_{2}$.

An analogous method has been used here; the distribution of thorium between benzene and water has been measured as a function of the concentration of free $\mathrm{Aa}^{-}$in the aqueous phase. The radioactive isotope $\mathrm{Th}^{234}$ $\left(\mathrm{UX}_{1}\right)$ was used in tracer amounts, never exceeding $10^{-10} \mathrm{~mol} / \mathrm{l}$.

By the use of radioactive tracers instead of macro amounts several advantages are gained: a) the ligand concentration can be increased without exceeding the solubility product of the uncharged complex, b) the concentrations of other substances in solution are not affected by the addition of tracer amounts of the central atom; this will simplify the mathematical treatment, and c) in general the radiometric method of analysis is much simpler than other analytical methods. Grahame and Seaborg ${ }^{8}$ have shown that the distribution ratio of tracer isotopes between two phases is equal to that for macro amounts of the same element.

Zirconium gives a complex with thenoyltrifluoracetone (TTA) soluble in benzene. Connick and $\mathrm{McVey}{ }^{(20)}$ have investigated the distribution of radioactive $\mathrm{Zr}^{95}$ between benzene and water, in which some TTA had been dissolved, as a function of the concentration of different organic and inorganic anions. From these measurements they could calculate some complex formation constants. This method is an extension of that used by Morse, and 
may in the future be of great value for determining complexes where emf and optical methods fail. However the constants obtained by these authors should not be regarded as definite until some of the assumptions made concerning the system zirconium-TTA-water have proved to be true.

\section{DEPENDENCE OF THE DISTRIBUTION COEFFICIENT ON THE HAa CONCENTRATION}

Many authors have given general mathematical treatments of the formation of mononuclear ${ }^{\text {e.g. 3,9 }}$ and polynuclear e.g. 4,9,10 complexes. The discussion will treat only the special case of thorium complexes in aqueous. solution.

It is presumed that no ions exist in the benzene phase. In the following the index aq and the charges of ions are omitted for the sake of simplicity (E. g. $\left[\mathrm{ThAa}_{2}\right]$ means the concentration of the ion $\mathrm{ThAa}_{2}^{2+}$ in the aqueous phase). Since all solutions have an approximately constant ionic strength of 0.01 , all activity coefficients are constant, and by using concentrations in the law of mass action, stoichiometric constants are obtained.

In an aqueous solution of thorium and acetylacetone mononuclear complexes $\mathrm{ThAa}_{n}$ and polynuclear complexes $\mathrm{Th}_{m} \mathrm{Aa}_{n}$ might occur. Moreover we have products of hydrolysis, which can be written as $\mathrm{Th}(\mathrm{OH})_{n}, \ldots \ldots$ $\mathrm{Th}_{m}(\mathrm{OH})_{n}$, and mixed complexes $\mathrm{Th}_{m} \mathrm{Aa}_{n}(\mathrm{OH})_{p}$. If hydrolysis can be neglected, mixed complexes can probably also be neglected. Indeed, no evidence for their existence was obtained, and no mathematical treatment for mixed complexes will be made here. The following section I will deal with the simplest case, when only mononuclear complexes are formed. In II the general case is treated.

I. Mononuclear complexes are formed

We define the complex product $x_{n}$ by

$$
x_{n}=\left[\mathrm{ThAa}_{n}\right][\mathrm{Th}]^{-1}[\mathrm{Aa}]^{-n}
$$

If the hydrolysis product, $\beta_{n}$, is defined analogous to the complex product, we have

$$
\beta_{n}=\left[\mathrm{Th}(\mathrm{OH})_{n}\right][\mathrm{Th}]^{-1}[\mathrm{OH}]^{-n}
$$

The total concentration of thorium in the aqueous phase [Th $]_{\text {aq, tot }}$ must then be 


$$
[\mathrm{Th}]_{\mathrm{aq}, \text { tot }}=[\mathrm{Th}]+\sum_{n=1}^{n=N}\left[\mathrm{ThAa}_{n}\right]+\sum_{n=1}^{n=P}\left[\mathrm{Th}(\mathrm{OH})_{n}\right]
$$

$\mathrm{ThAa}_{N}$ and $\mathrm{Th}(\mathrm{OH})_{p}$ are the complexes with maximum number ligands respectively. Now the measurements are made on the radioactive $\mathrm{Th}{ }^{234}$ in solution. The radioactivity in the aqueous phase, $I_{\mathrm{aq}}$ disintegrations/ minute, under defined conditions must be proportional to [Th $)_{\mathrm{aq}, \text { tot }}$ :

$$
[\mathrm{Th}]_{\mathrm{aq}, \text { tot }}=\Lambda_{\mathrm{aq}} \cdot I_{\mathrm{aq}}
$$

The only existing form of thorium in the benzene phase at these low concentrations is $\mathrm{ThAa}_{4}$. At macro concentrations some association between $\mathrm{ThAa}_{4}$ molecules takes place; the evidence for this is apparent from the studies of Biltz and Clinch ${ }^{11}$. Using the subscript be for the benzene phase, we have

$$
[\mathrm{Th}]_{\mathrm{be}, \text { tot }}=\left[\mathrm{ThAa}_{4}\right]_{\mathrm{be}}
$$

If the distribution factor of $\mathrm{ThAa}_{4}$ is $\lambda_{4}$,

we get

$$
\lambda_{4}=\left[\mathrm{ThAa}_{\mathbf{4}}\right]_{\mathrm{be}} /\left[\mathrm{ThAa}_{\mathbf{4}}\right]_{\mathrm{aq}}
$$

$$
[\mathrm{Th}]_{\mathrm{be}, \text { tot }}=\lambda_{4}\left[\mathrm{ThAa}_{4}\right]_{\mathrm{aq}}
$$

If the measurements on the benzene phase are also made under defined conditions we obtain

$$
[\mathrm{Th}]_{\mathrm{be}, \text { tot }}=\Lambda_{\mathrm{be}} \cdot I_{\mathrm{be}}
$$

Now we may define the apparent distribution coefficient $q$ :

$$
q=I_{\mathrm{be}} / I_{\mathrm{aq}}=[\mathrm{Th}]_{\mathrm{be}, \text { tot }} \cdot \Lambda_{\mathrm{aq}} /[\mathrm{Th}]_{\mathrm{aq}, \text { tot }} \cdot \Lambda_{\mathrm{be}}
$$

We define $\lambda_{4}^{\prime}$ by

$$
\lambda_{4}^{\prime}=\lambda_{4} \cdot \Lambda_{\mathrm{aq}} / \Lambda_{\mathrm{be}}
$$

It will be shown later that $\Lambda_{\mathrm{be}} / \Lambda_{\mathrm{aq}}$ is approximately 1 in the present case. With Equations (1) - (9) we get

$$
q=\frac{\lambda_{4}^{\prime} x_{4}[\mathrm{Aa}]^{4}}{1+\sum x_{n}[\mathrm{Aa}]^{n}+\sum \beta_{n}[\mathrm{OH}]^{n}}
$$


This equation shows that different curves $q=f$ [Aa] will be obtained for different $\mathrm{pH}$, if $\sum \beta_{n}[\mathrm{OH}]^{n}$ cannot be neglected when compared with $1+\sum x_{n}[\mathrm{Aa}]^{n}$. If the hydrolysis is negligible, Equation (10a) will take the form (note that $x_{0}=1$ )

$$
q=\frac{\lambda_{4}^{\prime} \cdot x_{4}[\mathrm{Aa}]^{4}}{\sum_{n=0}^{n=N} x_{n}[\mathrm{Aa}]^{n}}
$$

If we introduce a set of new constants $\varphi_{n}$, where

$$
\varphi_{4-n}=\left[\operatorname{ThAa}_{n}\right]\left[\operatorname{ThAa}_{4}\right]^{-1}[\mathrm{Aa}]^{4-n}=x_{n} \cdot x_{4}^{-1}
$$

Equation (10b) can be simplified to

$$
q=\lambda_{4}^{\prime}\left(\sum \varphi_{4-n}[\mathrm{Aa}]^{n-4}\right)^{-1}
$$

We have $\varphi_{0}=x_{0}=1$. If no negatively charged complexes are formed, then

$$
q=\frac{\lambda_{4}^{\prime}}{\varphi_{4}[\mathrm{Aa}]^{-4}+\varphi_{3}[\mathrm{Aa}]^{-3}+\varphi_{2}[\mathrm{Aa}]^{-2}+\varphi_{1}[\mathrm{Aa}]^{-1}+1}
$$

In case negative complexes $\mathrm{ThAa}_{5}^{-}, \mathrm{ThAa}_{6}^{2-}$, etc., are formed additional terms $\varphi_{-1}[\mathrm{Aa}], \varphi_{-2}[\mathrm{Aa}]^{2}$, etc., occur in the denominator of Equation (12b).

Acetylacetone is a weak acid with the dissociation constant $K_{a}=1.17 \cdot 10^{-9}$ at $25^{\circ} \mathrm{C}^{(12)}$. If $V_{\text {be }}^{\circ} \mathrm{ml}$ benzene with an acetylacetone concentration of [HAa] ${ }_{\text {be }}^{\circ}$ is shaken with $V_{\text {aq }}^{\circ} \mathrm{ml}$ water containing no acetylacetone, we find after equilibrium that

$$
[\mathrm{HAa}]_{\mathrm{aq}}=[\mathrm{HAa}]_{\mathrm{be}}^{\circ}\left(1+K_{a}\left[\mathrm{H}^{+}\right]^{-1}+D V_{\mathrm{be}} / V_{\mathrm{aq}}\right) \cdot V_{\mathrm{aq}} / V_{\mathrm{be}}
$$

where $D$ is the distribution coefficient of acetylacetone between the two liquids. $D$ is practically constant for $\mathrm{pH}$ between 1 and 7 , and an ionic strength less than $0.02 \mathrm{~mol} / 1 ; D$ was found to be $5.95^{(13)}$. $V_{\mathrm{aq}}$ and $V_{\mathrm{be}}$ are the equilibrium volumes of the two phases; in this case we have assumed $V_{\mathrm{aq}}^{\circ}=V_{\mathrm{aq}}$ and $V_{\mathrm{be}}^{\circ}=V_{\mathrm{be}}$, which is true for low acetylacetone concentrations. If $\mathrm{pAa}=$ $-\log \left[\mathrm{Aa}^{-}\right]$and $V_{\text {aq }}=V_{\text {be }}$ we have

$$
\mathrm{pAa}=\mathrm{p} K_{a}-\mathrm{pH}-\log [\mathrm{HAa}]_{\mathrm{be}}^{\circ} /\left(D+1+\left[\mathrm{H}^{+}\right]^{-1} K_{a}\right)
$$


For $1<\mathrm{pH}<7$ we can in the case of acetylacetone neglect $\left[\mathrm{H}^{+}\right] . K_{a}$ when compared to $D+1$. Introducing the values of the constants we get

$$
\mathrm{pAa}=9.77-\mathrm{pH}-\log [\mathrm{HAa}]_{\mathrm{be}}^{\circ}
$$

The addition of tracer amounts of thorium to the acetylacetone solution will bind some $\mathrm{Aa}^{-}$, but this amount is negligible when compared to the amount of acetylacetone in the two phases.

II. Polynuclear complexes are formed

If complexes with two or more central atoms are formed the complex product must be defined by

$$
x_{m, n}=\left[\mathrm{Th}_{m} \mathrm{Aa}_{n}\right][\mathrm{Th}]^{-m}[\mathrm{Aa}]^{-n}
$$

in which $x_{1, n}=x_{n}$ from Equation (1). For the analogous hydrolysis constants $\beta_{m, n}$ can be defined. Since Equations (4) - (9) are still valid, we get

$$
q=\frac{\lambda_{4}^{\prime} x_{4}[\mathrm{Aa}]^{4}}{1+\sum \sum m \beta_{m, n}[\mathrm{Th}]^{m-1}[\mathrm{OH}]^{n}+\sum \sum m x_{m, n}[\mathrm{Th}]^{m-1}[\mathrm{Aa}]^{n}}
$$

In this equation the apparent distribution coefficient $q$ is a function of not only $\mathrm{pH}$ and [Aa] but also of the thorium concentration [Th]. It would be a difficult task to determine the constants $x_{m, n}$ and $\beta_{m, n}$ in this general case; however under the experimental conditions of the present work, case I seems to be realised, as might have been expected because of the very low thorium concentration.

\section{EXPERIMENTAL}

Chemicals used

Except for thorium and acetylacetone all chemicals employed were of analytical grade and were used without further purification or analysis.

Pure acetylacetone was prepared according to a method described elsewhere ${ }^{13}$. It was kept in benzene solution.

The thorium isotope $\mathrm{Th}^{234}\left(\mathrm{UX}_{1}\right)$ is a natural disintegration product of $\mathrm{U}^{238}$ (UI), the most common uranium isotope. The uranium now produced for use in reactors contains no other beta active thorium isotope than $\mathrm{Th}^{234}$ and $\mathrm{Th}^{231}$ (UY), which is a disintegration product of $\mathrm{U}^{235}$, and disintegrates by soft $\beta$-emission. When radioactive equilibrium exists between UI and $U X_{1}, 1 \mathrm{mg}$ of uranium will give 738 beta particles per minute from $\mathrm{UX}_{1}$. The energy of these beta particles is too low $(0.12 \mathrm{MeV})$ to be mea- 
sured by common glass-wall GM-counters. However the next member in the disintegration series $\mathrm{UX}_{2}\left(\mathrm{~Pa}^{234}\right)$ has a beta energy of $2.32 \mathrm{MeV}$, and this high energy makes it easily detectable. Radioactive equilibrium between $\mathrm{UX}_{1}$ and $\mathrm{UX}_{2}$ will be practically reached within 10 minutes; after this time the radioactivity of $\mathrm{UX}_{2}$ indicates where $U X_{1}$ is to be found. The half-life of $U X_{1}$ is 24.1 days ${ }^{21}$.

Dyrssen 14 has given a fairly simple method for preparing carrier-free $U X_{1}$. A few grams of uranyl nitrate are shaken with some $10 \mathrm{ml}$ of ether, previously saturated with water. In the small aqueous layer formed, practically all $\mathrm{UX}_{1}$ is to be found. This aquous phase is diluted with a few $\mathrm{ml}$ of $0.1 \mathrm{M} \mathrm{HCl}$ and run through ani on exchanger column (Wofatit $\mathrm{KS}$ ): $\mathrm{Th}^{4+}\left(\mathrm{UX}_{1}\right)$ and $\mathrm{UO}_{2}^{2+}$ are adsorbed. The uranyl ions are washed out with $2 M \mathrm{HCl}$. UX $\mathrm{UX}_{1}$ is desorbed by $0.5 M$ oxalic acid, and the eluate is evaporated. The oxalic acid then sublimes, leaving carrier free $\mathrm{UX}_{1}$, which is dissolved in a small amount of strong $\mathrm{HClO}_{4}$.

\section{Distribution experiments}

The two phases were prepared in a $60 \mathrm{ml}$ separatory funnel in the following way:

To $0.03 \mathrm{ml}$ of $\mathrm{UX}_{1}$ solution, calculated amounts of water, $\mathrm{HClO}_{4}$ and $\mathrm{NaOH}$ were added so as to give $15 \mathrm{ml}$ solution of ionic strength 0.01 and the acidity desired. Finally $15 \mathrm{ml}$ of benzene of a known acetylacetone concentration, [HAa] ${ }^{\circ}$ be, was added.

The separatory funnels were sealed with glass stoppers and shaken for about 20 hours at $25.0 \pm 0.2^{\circ} \mathrm{C}$. The loss of solution due to possible leakage was less than $0.1 \%$. The phases were allowed to separate in the thermostat, definite volumes of both phases were taken out, and their radioactivity $I_{\mathrm{aq}}$ and $I_{\mathrm{be}}$ was measured in a reproducible manner (see next section).

The $\mathrm{pH}$ of the aqueous phase was measured with a glass electrode and a standard pH-meter; the accuracy was $\pm 0.02 \mathrm{pH}$-units. From the amount of $\mathrm{HClO}_{4}$ and $\mathrm{NaOH}$ added, and [HAa] ${ }^{\circ}$, the $\mathrm{pH}$ could be roughly predicted for $\mathrm{pH} \leq 4$, but not for higher $\mathrm{pH}$. No buffer solutions were added since these might give complexes with $\mathrm{Th}^{4+}$ and thus compete with the acetylacetone; no complexes are formed between sodium and acetylacetone ${ }^{15,16 .}$

Theradioactive measurements

The radioactivity of $\mathrm{UX}_{1}$ (or actually $\mathrm{UX}_{2}$ ) could be measured either in the solution directly or after evaporation to dryness. The first method was found to be the most convenient.

The radioactive liquid to be measured is contained in a special "immersion cup". The arrangement with the GM-tube (Type B6, 20th Century Electronics, London; wall thickness about $30 \mathrm{mg} / \mathrm{cm}^{2}$ ) is shown in Fig. 1 . When inserting the GM-tube in the immersion cup, liquid is pressed up and surrounds the sensitive part of the counter tube. The GMtube is well centered in the cup, but separate experiments have shown that this is not too important. This is also to be expected since the mean range of beta particles from $\mathrm{UX}_{2}$ is $3 \mathrm{~mm}$ in water, while the thickness of the liquid layer is about $1 \mathrm{~mm}$.

On each cup a line has been etched. When liquid is filled to this line and the GMtube is inserted, the liquid surface will reach the same height for different cups. As these cups are not exactly of the same internal diameter $( \pm 0.2 \mathrm{~mm}$ ) different cups will contain different volumes of liquid. In the experiment recorded in Fig. 2, different volumes of a solution of constant specific radioactivity were brought into a certain immersion cup, and the number of counts per minute, $I$, measured. On this cup the line was etched 


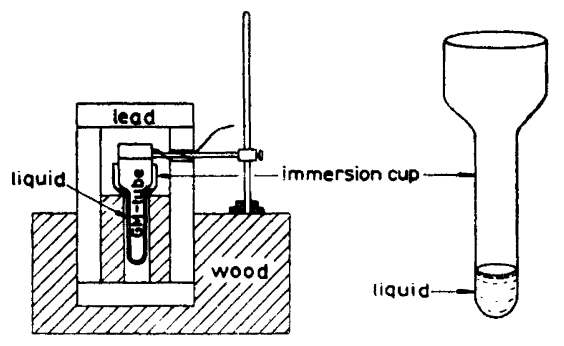

Fig. 1. The experimental arrangement with the GM-tube inserted in the immersion cup.

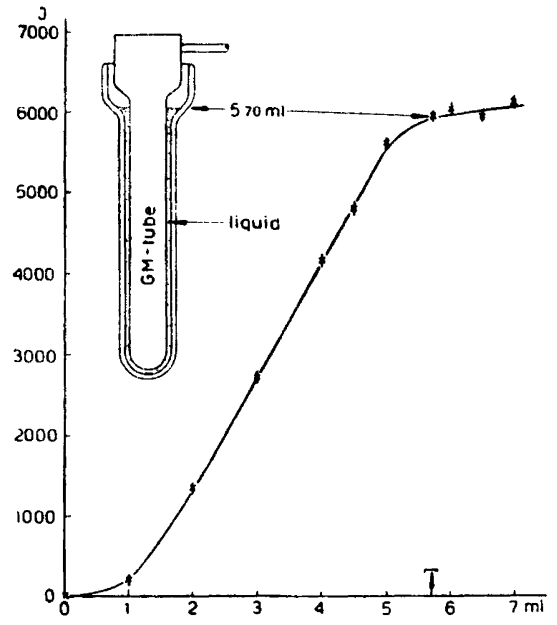

Fig. 2. The variation of radioactivity measured (I counts/minute) as a function of the volume in $m l$ of an $U X_{1}$-solution of constant specific activity.

\section{$8 \mathrm{xt}$}

at $5.7 \mathrm{ml}$, where a variation of $\pm 0.1 \mathrm{ml}$ will change $I$ by less than $1 \%$. Due to the different volumes of liquid in the cups these have been calibrated against each other with a standard $\mathrm{UX}_{1}$-solution; this introduces the calibration factor, $\psi$. The $\psi$-scale was arbitrarily chosen by making $\psi=1$ for one cup, the "standard immersion cup".

Before the measurements on $U_{X_{1}}$ the cup is filled with pure water and the zero effect $I_{0}$ is determined. If the following measurement on the aqueous $\mathrm{UX}_{\mathbf{1}}$-solution gives $I_{m}$, we have the corrected activity $I_{\mathrm{aq}}$ :

$$
I_{\mathrm{aq}}=\psi\left(I_{m}-I_{0}\right)
$$

For the activity in the benzene phase an analogous equation is obtained; in this case the zero effect is taken on pure benzene. The radioactivity adsorbed on the glass wall of the GM-tube is desorbed by washing with $\mathrm{H}_{2} \mathrm{SO}_{4}-\mathrm{K}_{2} \mathrm{Cr}_{2} \mathrm{O}_{7}$.

The deviations given in Table 1 are standard errors (the square root ot the total number of counts divided by the time of measurement). Only in one case the activity was so high, that corrections for the resolving time of the counter had to be made.

To determine the efficiency of the counting arrangement an immersion cup was filled with a known amount of uranyl nitrate solution, and the radioactivity was measured; this gave $131 \pm 1$ counts $/ \mathrm{min}$. $\mathrm{mg} U$. It is known that $1 \mathrm{mg} \mathrm{U}$ in equilibrium with $\mathrm{UX}_{1}$ and $\mathrm{UX}_{2}$ gives 738 beta $\mathrm{UX}_{2} / \mathrm{min}$. From these two values we calculate that $131 / 738=$ $17.8 \%$ of all betas from $\mathrm{UX}_{2}$ in the solution are counted by the electronic circuit.

From $1 \mathrm{~g}$ uranium, which was completely freed from thorium one year ago, there will now be about $7.2 \cdot 10^{-13} \mathrm{~mol} \mathrm{Th}^{230}$ (Io) $+\mathrm{Th}^{234}$. This amount of Th will give approximately $7.4 \cdot 10^{5} \beta$ disintegrations/minute. We can now find for the standard immersion 
cup, which has a $\psi=1$ and a volume of $5.20 \mathrm{ml}$, that 1 count $/ \mathrm{min}$ corresponds to $7.2 \cdot 10^{-13} / 7.4 \cdot 10^{5} \cdot 5.20 \cdot 10^{-3} \cdot 0.178=1.05 \cdot 10^{-15} \mathrm{~mol} \mathrm{Th} / \mathrm{l}$. According to Equation (4a) this value must be $\Lambda_{\mathrm{aq}}$; thus we have

$$
[\mathrm{Th}]_{\mathrm{aq}, \text { tot }}=1.05 \cdot 10^{-15} \cdot I_{\mathrm{aq}} \mathrm{mol} / \mathrm{l}
$$

The absorption of beta particles varies with the density of the absorber e.g. (17). The exponential relationship $I=I_{0} \cdot e^{-u x}$, where $x$ is the thickness in cm of the absorbing layer and $u$ is the absorption coefficient, is roughly valid for beta particles. The mass absorption coefficient $u / d$, where $d$ is the density, is nearly independent of the atomic number of the absorber. If $E_{m}$ is the maximum energy of the betas, one can use the empirical relationship $E_{m}^{1.33} \cdot u=22 . d$ to find $u$. For $E_{m}=2.32 \mathrm{MeV}$ and $d_{\text {be }}=0.897$ we can calculate $I / I_{0}=0.697$ for a water layer of $0.5 \mathrm{~mm}$, and 0.729 for a benzene layer of the same thickness. This means that the absorption of beta particles from $\mathrm{UX}_{2}$ in $0.5 \mathrm{~mm}$ of water is $0.729 / 0.697$ times that in benzene. If the experimental conditions are the same for the measurements on the benzene solution to those for the aqueous solution, we have $\Lambda_{\mathrm{be}}=\Lambda_{\mathrm{aq}} \cdot 0.697 / 0.729$, or $\Lambda_{\mathrm{be}}=1.00 \cdot 10^{-15} \mathrm{~mol} . \mathrm{min} / 1$. counts. Thus we have

$$
[\mathrm{Th}]_{\text {be, tot }}=1.00 \cdot 10^{-15} \cdot I_{\text {be }} \mathrm{mol} / \mathrm{l}
$$

\section{ANALYSIS OF THE DISTRIBUTION CURVE}

In Table 1 the experimental data ([HAa $\left.]_{\mathrm{be}}^{\circ}, \mathrm{pH}, I_{\mathrm{be}}, I_{\mathrm{aq}}\right)$ are tabulated. From $\mathrm{pH}$ and $[\mathrm{HAa}]_{\mathrm{be}}^{\circ}$ we can calculate $[\mathrm{HAa}]_{\mathrm{aq}}$ and $\mathrm{pAa}$ by means of Equation (15). With Equation (8) $I_{\text {be }}$ and $I_{\text {aq }}$ gives $q$. The symbols in the last column of Table 1 are the same as shown in Fig. 3, where $\log q$ is given as a function of pAa (this curve will be referred to as the "distribution curve"). Table 1 is divided in five sections, each representing a definite $\mathrm{pH}-$ range.

In Fig. 3 almost all points are on one continuous curve. For the five symbols $\#$ and $\Delta$ the concentration of acetylacetone in the benzene phase is 5-molar; $i . e$. the benzene solution consists of $50 \%$ acetylacetone. The $q$ measured for these points is not the distribution of $U X_{1}$ between benzene and water, but the distribution between $50 \%$ benzene $+50 \%$ acetylacetone and water. Thus the low values of $q$ obtained here are either due to that $\mathrm{ThAa}_{4}$ is more soluble in benzene than in acetylacetone, which seems improbable, or that $\mathrm{ThAa}_{4}$ is less soluble in pure water than in water containing much acetylacetone. These five $q$ values have not been used in the calculation of the complex constants.

In the deduction of Equation (10a) it was pointed out that if the thorium hydrolysis influences the equilibrium between thorium and acetylacetone, different curves $q=f[\mathrm{Aa}]$ should be obtained for different $\mathrm{pH}$. At $\mathrm{pH}>3$ the thorium hydrolysis is considerable ${ }^{18}$. As the $\mathrm{pH}$ was widely different 


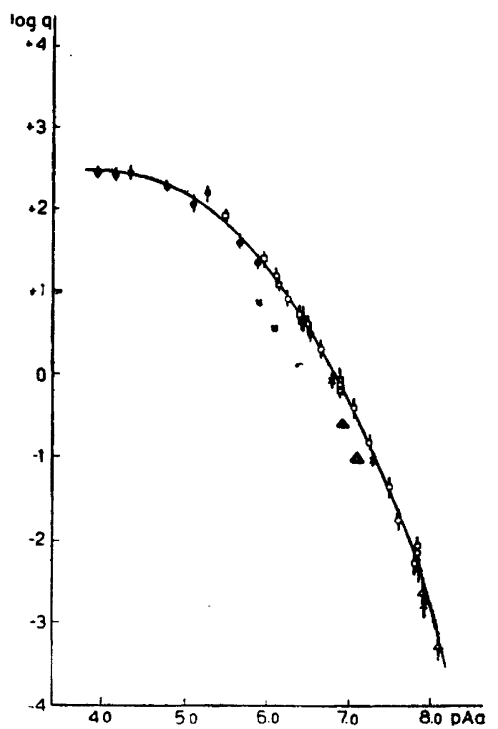

Fig. 3. The distribution of $U X_{1}$ between benzene and water $(q)$ as a function of the concentration of acetylacetonate ions $(p A a=$ $-\log \left[\mathrm{Aa}^{-}\right]$. The different signs relate to $\mathrm{pH}$ values according to Table $1: \Delta p H \approx 2$; $\times p H \approx 3 ; \quad \bigcirc p H \approx 4 ;$ 口 $p H \approx 5$; $p H \approx 6$. The curve was drawn to fit the experimental points; it was used for calculating $x_{n}$ by method $A$.

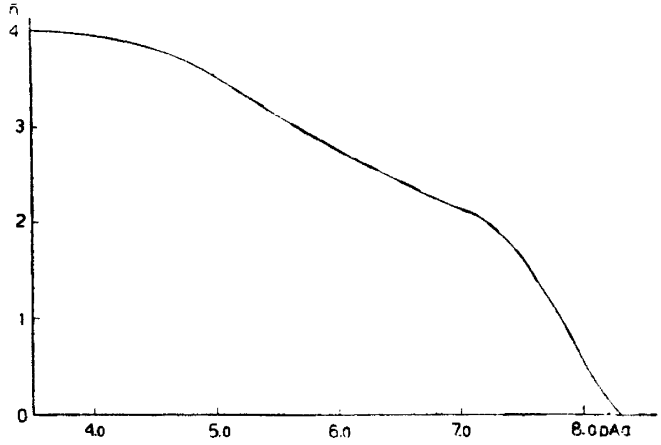

Fig. 4. The average number of ligands per central atom $(\bar{n})$ as a function of the concentration of free ligand ( $p A a=-l o g$ $\left[\mathrm{Aa}^{-}\right]$), calculated from the smooth curve in Figure 3.

in the five series of measurement (see Table 1) the curvature in Fig. 3 can not be due to the thorium hydrolysis, but solely to the complex formation between thorium and acetylacetone.

It is shown in Equation (17) that at constant $\mathrm{pH}$ and $\mathrm{pAa}, q$ will be a function of [Th] if polynuclear complexes are formed. A separate investigation at $\mathrm{pH} 3$ and $\mathrm{pAa} 6.9$ showed that a variation of $I_{\mathrm{aq}}$ from 10 to over 1,000 counts/min had no influence on $q$, which was about 1 . It is thus concluded that no polynuclear complexes are formed at these low concentrations.

\section{THE CALCULATION OF COMPLEX CONSTANTS FROM THE DISTRIBUTION CURVE}

It is clear from the considerations in the preceeding section that hydrolysis and polynuclear complexes can be neglected; then Equation (10b) is valid. There are many ways to evaluate the constants in this equation; here two independent methods will be used. 
A. The ligand number $\bar{n}$ is generally defined as the mean number of ligands per central atom. In our case we have

$$
\bar{n}=\sum n\left[\operatorname{ThAa}_{n}\right]_{\mathrm{aq}} /[\mathrm{Th}]_{\mathrm{aq}, \text { tot }}
$$

The fraction of Th present in form of the $n^{\text {th }}$ complex is

$$
\alpha_{n}=\left[\operatorname{ThAa}_{n}\right]_{\mathrm{aq}} /[\mathrm{Th}]_{\mathrm{aq}, \text { tot }}
$$

Comparing this with Equations (6) - (9) we find that

$$
q=\alpha_{4} \cdot \lambda_{4}^{\prime}
$$

From Equations (21) and (22) we can deduce

$$
\bar{n}=n+d\left(\log \alpha_{n}\right) / d(\mathrm{pAa})
$$

which gives together with (23)

$$
\bar{n}=4+d(\log q) /(d \mathrm{pAa})
$$

Fig. 3 shows $\log q$ as a function of pAa. By graphical derivation of this curve using Equation (25) we can obtain $\bar{n}=f(\mathrm{pAa})$, the complex formation curve, which is shown in Fig. 4. We transform Equation (21) to a form more suitable for mathematical treatment ${ }^{(3)}$ :

$$
\begin{gathered}
\bar{n}+(\bar{n}-1) \varkappa_{1}[\mathrm{Aa}]+(\bar{n}-2) \varkappa_{2}[\mathrm{Aa}]^{2}+(\bar{n}-3) \varkappa_{3}[\mathrm{Aa}]^{3}+ \\
+(\bar{n}-4) \varkappa_{4}[\mathrm{Aa}]^{4}=0
\end{gathered}
$$

Each point on the curve in Fig. 4 will give an equation of this kind. Four points on the curve will suffice for the determination of all the constants; we take $\vec{n}=0.5,1.5,2.5$ and 3.5. The solution of the set of equations thus obtained is achieved by using determinants. From these values of $x_{n}$ we use Equation (11) to calculate $\varphi_{n}$, and thus obtain the following values:

$$
\varphi_{1}=1.13 \cdot 10^{-5}, \quad \varphi_{2}=4.38 \cdot 10^{-12}, \quad \varphi_{3}=1.63 \cdot 10^{-19}, \quad \varphi_{4}=1 \cdot 15 \cdot 10^{-27} .
$$

The values $\bar{n}=n-0.5$ are taken because at each of these points two complexes dominate, and the errors from the presence of the other complexes are negligible. Consequently we find 


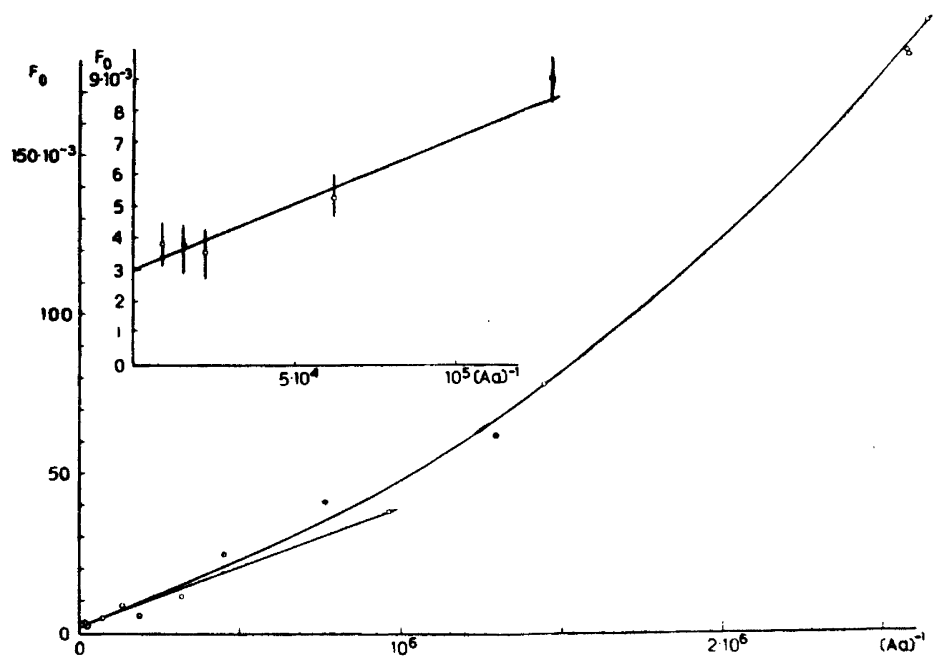

Fig. 5. The variation of $F_{0}=1 / q$ with $\left[\mathrm{Aa}^{-}\right]^{-1}$ calculated from the experimental points in Figure 3. The intercept on the $F_{0}$-axis gives $1 / \lambda_{4}^{\prime}$ and the slope of the curve at this point is $\varphi_{1} / \gamma_{4}^{\prime}$.

$$
[\mathrm{Aa}]_{\bar{n}}^{-1}=n-0.5 \approx x_{n} x_{n-1}^{-}=k_{n}
$$

where $k_{n}$ is called the complex formation constant:

$$
k_{n}=\left[\mathrm{ThAa}_{n}\right]\left[\mathrm{ThAa}_{n-1}\right]^{-1}[\mathrm{Aa}]^{-1}
$$

B. Before proceeding to calculate the complex formation constants, we will see what values of $\varphi_{n}$ will be obtained by the second method, which is analogous to that used by Leden ${ }^{9}$; with this method the influence of the experimental errors is more clearly seen, and the uncertain procedure of taking derivatives on a smoothed curve is avoided.

Equation (12b) is transformed to

$$
F_{0}=1 / q=\left(1+\varphi_{1}[\mathrm{Aa}]^{-1}+\varphi_{2}[\mathrm{Aa}]^{-2}+\varphi_{3}[\mathrm{Aa}]^{-3}+\varphi_{4}[\mathrm{Aa}]^{-4}\right) \lambda_{4}^{\prime-1}
$$

In Table $2 F_{0}$ and $[\mathrm{Aa}]^{-1}$ are calculated from the data in Table 1 (not from the smooth curve in Fig. 3). In Fig. $5 F_{0}$ is given as a function of $[\mathrm{Aa}]^{-1}$. The intercept on the $F_{0}$-axis gives $1 / \lambda_{4}^{\prime}$ and the slope of the curve at this point gives $\varphi_{1} / \lambda_{4}^{\prime}$ according to Equation (29). We then get $\lambda_{4}^{\prime}=330 \pm 30$ and $\varphi_{1}=(1.2 \pm 0.2) 10^{-5}$. 


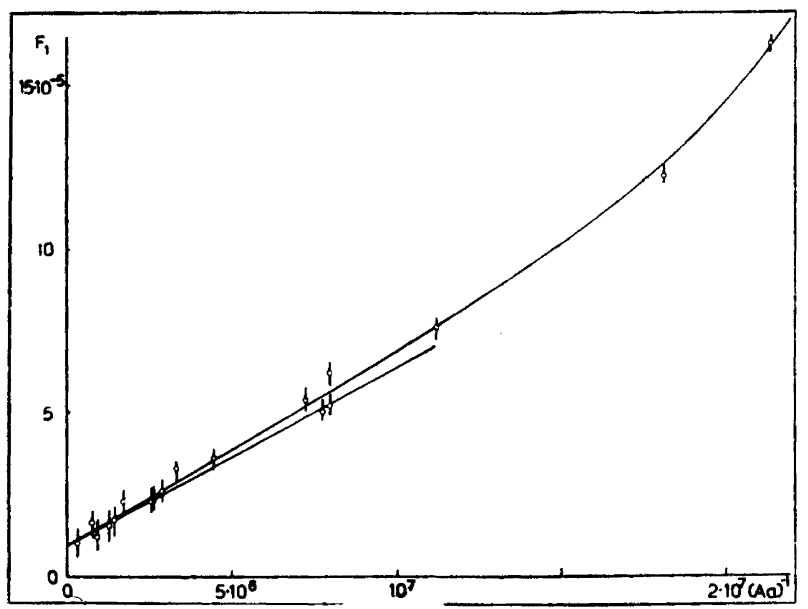

Fig. 6. F $F_{1}$ calculated according to Equation (30) as a function of $\left[\mathrm{Aa}^{-}\right]^{-1}$. The intercept on the $F_{1}$-axis gives $\varphi_{1}$ and the slope of the curve at this point $\varphi_{2}$.

In an analogous manner we derive functions $F_{1}, F_{2}$, etc.,

$$
\begin{gathered}
F_{1}=\left(\frac{\lambda_{4}^{\prime}}{q}-1\right)[\mathrm{Aa}]=\varphi_{1}+\varphi_{2}[\mathrm{Aa}]^{-1}+\varphi_{3}[\mathrm{Aa}]^{-2}+\varphi_{4}[\mathrm{Aa}]^{-3} \\
F_{2}=\left(F_{1}-\varphi_{1}\right)[\mathrm{Aa}]=\varphi_{2}+\varphi_{3}[\mathrm{Aa}]^{-1}+\varphi_{4}[\mathrm{Aa}]^{-2}
\end{gathered}
$$

The values of $F_{1}$ and $F_{2}$ are found in Table 2. In Figs. 6 and 7 they are given as functions of $[\mathrm{Aa}]^{-1}$. From Fig. 6 we obtain $\varphi_{1}=(1.0 \pm 0.1) 10^{-5}$ and $\varphi_{2}=(5.4 \pm 0.3) 10^{-12}$. This $\dot{\varphi}_{1}$ is somewhat lower than the $\varphi_{1}$ obtained from Fig. 5, but as it is more accurate it has been used for the calculation of $F_{2}$. From Fig. 7 we have $\varphi_{2}=(5 \pm 1) 10^{-12}$; no calculation of $F_{3}$ has been done because the errors will be to great. As the value of $\varphi_{2}$ from Fig. 6 is more exact than that from Fig. 7 , the value $\varphi_{2}=(5.4 \pm 0.3) 10^{-12}$ has been accepted.

As the errors in the calculation of $F_{2}$ are considerable, no attempt to find $F_{3}$ and $F_{4}$ has been done. To obtain $\varphi_{3}$ and $\varphi_{4}$ we deduce

$$
F_{5}=\left(\frac{\lambda_{4}^{\prime}}{q}-1\right)[\mathrm{Aa}]^{4}=\varphi_{4}+\varphi_{3}[\mathrm{Aa}]+\varphi_{2}[\mathrm{Aa}]^{2}+\varphi_{1}[\mathrm{Aa}]^{3}
$$

$F_{5}$ is calculated in Table 2 and given as a function of [Aa] in Fig. 8. From this curve we find $\varphi_{4}=(1.5 \pm 0.5) 10^{-27}$ and $\varphi_{3}=(1.0 \pm 0.2) 10^{-19}$. 
Table 1. Calculation of the distribution curve from experimental data.

\begin{tabular}{|c|c|c|c|c|c|c|c|c|}
\hline $\mathrm{pH}$ & {$[\mathrm{HAa}]_{\mathrm{be}}^{0}$} & {$[\mathrm{HAa}]_{\mathrm{aq}}$} & pAa & $I_{\mathrm{be}}$ & $I_{\mathrm{aq}}$ & $q$ & $\log q$ & \\
\hline 1.90 & 0.605 & 0.087 & 8.10 & $10 \pm 1$ & 18200 & 0.00055 & -3.26 & $\Delta$ \\
\hline 1.98 & 6.73 & 0.967 & 6.97 & 282 & 1179 & 0.240 & -0.62 & $\Lambda$ \\
\hline 1.98 & 5.38 & 0.775 & 7.07 & 81 & 826 & 0.098 & -1.01 & $\bar{\Lambda}$ \\
\hline 2.00 & 0.865 & 0.124 & 7.85 & $6.7 \pm 3$ & 1510 & 0.0044 & -2.30 & $\triangle$ \\
\hline 1.94 & 0.865 & 0.124 & 7.89 & $2.7 \pm 1$ & 1106 & 0.0024 & -2.70 & $\triangle$ \\
\hline 3.06 & 6.73 & 0.967 & 5.89 & 1030 & 143 & 7.20 & +0.86 & H \\
\hline 2.88 & 6.73 & 0.967 & 6.07 & 1290 & 359 & 3.59 & +0.56 & 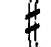 \\
\hline 2.66 & 5.38 & 0.775 & 6.39 & 750 & 595 & 1.26 & +0.10 & H \\
\hline 2.99 & 0.865 & 0.124 & 6.86 & 429 & 511 & 0.84 & -0.08 & $x$ \\
\hline 2.98 & 0.288 & 0.0415 & 7.33 & $81 \pm 5$ & 856 & 0.095 & -1.02 & $x$ \\
\hline 3.20 & 0.0450 & 0.0065 & 7.93 & $1.6 \pm 1.2$ & 974 & 0.0016 & -2.70 & $x$ \\
\hline 3.74 & 0.865 & 0.124 & 6.11 & 1238 & $78 \pm 1$ & 16.0 & +1.20 & 0 \\
\hline 3.72 & 0.806 & 0.116 & 6.16 & 1253 & $99 \pm 8$ & 12.7 & +1.10 & 0 \\
\hline 3.90 & 0.450 & 0.0645 & 6.23 & 474 & $59 \pm 3$ & 8.03 & +0.90 & 0 \\
\hline 4.02 & 0.225 & 0.0323 & 6.41 & 468 & 86 & 5.45 & +0.74 & 0 \\
\hline 3.85 & 0.323 & 0.0465 & 6.42 & 1047 & 203 & 5.16 & +0.71 & 0 \\
\hline 3.86 & 0.288 & 0.0465 & 6.46 & 996 & 223 & 4.33 & +0.64 & 0 \\
\hline 4.00 & 0.134 & 0.0193 & 6.65 & 1120 & 560 & 2.00 & +0.30 & 0 \\
\hline 4.05 & 0.0673 & 0.0097 & 6.90 & 339 & 430 & 0.79 & -0.10 & 0 \\
\hline 3.90 & 0.0673 & 0.0097 & 7.05 & 290 & 752 & 0.386 & -0.41 & 0 \\
\hline 3.75 & 0.0578 & 0.0083 & 7.26 & $92 \pm 5$ & 617 & 0.149 & -0.82 & 0 \\
\hline 3.93 & 0.0225 & 0.00323 & 7.50 & $35 \pm 2$ & 814 & 0.043 & -1.37 & 0 \\
\hline 3.70 & 0.0278 & 0.00397 & 7.63 & $11 \pm 3$ & 635 & 0.0173 & -1.76 & 0 \\
\hline 4.00 & 0.0090 & 0.00129 & 7.83 & $4.3 \pm 2.0$ & 867 & 0.0050 & -2.30 & 0 \\
\hline 3.88 & 0.0092 & 0.00133 & 7.85 & $4.0 \pm 2.0$ & 471 & 0.0084 & -2.10 & $\mathrm{O}$ \\
\hline 3.98 & 0.0090 & 0.00129 & 7.85 & $5.7 \pm 2.0$ & 791 & 0.0072 & -2.15 & 0 \\
\hline 4.93 & 0.225 & 0.0323 & 5.50 & 1100 & $14 \pm 2$ & 79.0 & +1.90 & $\square$ \\
\hline 4.97 & 0.0673 & 0.0097 & 5.98 & 790 & $31 \pm 2$ & 25.7 & +1.41 & $\square$ \\
\hline 4.54 & 0.0673 & 0.0097 & 6.41 & 668 & 120 & 5.57 & +0.75 & $\square$ \\
\hline 4.54 & 0.0225 & 0.00323 & 6.89 & 282 & 333 & 0.848 & -0.07 & $\square$ \\
\hline 4.75 & 0.0134 & 0.00194 & 6.90 & 228 & 342 & 0.667 & -0.18 & ㅁ \\
\hline 5.92 & 0.865 & 0.124 & 3.93 & 2210 & $8.5 \pm 0.9$ & 260 & +2.42 & - \\
\hline 5.69 & 0.806 & 0.116 & 4.18 & 1432 & $5.4 \pm 2.0$ & 265 & +2.42 & 0 \\
\hline 6.62 & 0.0673 & 0.0097 & 4.33 & 915 & $3.2 \pm 1.8$ & 285 & +2.45 & 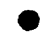 \\
\hline 6.64 & 0.0225 & 0.00323 & 4.79 & 689 & $3.6 \pm 1.5$ & 190 & +2.28 & - \\
\hline 5.84 & 0.0673 & 0.0097 & 5.11 & 720 & $6.7 \pm 3.0$ & 110 & +2.04 & 0 \\
\hline 5.68 & 0.0673 & 0.0097 & 5.27 & 1190 & $7.2 \pm 2$ & 165 & +2.18 & 0 \\
\hline 6.00 & 0.0134 & 0.00193 & 5.65 & 247 & $6.4 \pm 2$ & 38.6 & +1.59 & 0 \\
\hline 5.55 & 0.0225 & 0.00323 & 5.88 & 562 & $23 \pm 2$ & 24.4 & +1.38 & 0 \\
\hline 5.61 & 0.0045 & 0.00065 & 6.52 & 720 & 241 & 3.0 & +0.48 & - \\
\hline
\end{tabular}


Table 2. Calculation of $F_{n}$ from the data in Table 1 according to Equations (29)-(32). In Figures 5 to 8 these $F_{n}$ are used for the determination of $\varphi_{n}$.

\begin{tabular}{|c|c|c|c|c|c|}
\hline [Aa] & $1 /[\mathrm{Aa}]$ & $F_{0}$ & $F_{1}$ & $F_{2}$ & $F_{5}$ \\
\hline $11.8 \cdot 10^{-5}$ & $8.5 \cdot 10^{3}$ & 0.00385 & & & \\
\hline 6.62 & 15.1 & 0.00377 & . & & \\
\hline 4.67 & 21.4 & 0.00351 & & & \\
\hline 1.62 & 61.6 & 0.00526 & & & \\
\hline $78.0 \cdot 10^{-7}$ & $1.29 \cdot 10^{5}$ & 0.00909 & & & \\
\hline 53.8 & 1.86 & 0.00606 & & & \\
\hline 31.6 & 3.16 & 0.01266 & $10.04 \cdot 10^{-6}$ & & \\
\hline 22.4 & 4.47 & 0.02564 & 16.71 & & \\
\hline 13.2 & 7.59 & 0.04167 & 16.83 & & \\
\hline 10.46 & 9.56 & 0.03846 & 12.23 & $2.33 \cdot 10^{-12}$ & \\
\hline 7.75 & 12.9 & 0.06250 & 15.21 & 4.04 & \\
\hline 6.94 & 14.4 & 0.07874 & 17.34 & 5.09 & \\
\hline 5.88 & 17.0 & 0.1245 & 23.6 & 8.00 & \\
\hline 3.89 & 25.7 & 0.1828 & 23.1 & 5.10 & \\
\hline 3.89 & 25.7 & 0.1835 & 23.2 & 5.13 & \\
\hline 3.80 & 26.3 & 0.1938 & 23.9 & 5.28 & \\
\hline 3.47 & 28.8 & 0.2309 & 26.1 & 5.59 & \\
\hline 3.02 & 33.1 & 0.333 & 32.9 & 6.92 & \\
\hline 2.24 & 44.6 & 0.500 & 36.7 & 5.98 & $41.2 \cdot 10^{-26}$ \\
\hline 1.38 & 72.4 & 1.190 & 54.1 & 6.09 & 14.3 \\
\hline $12.89 \cdot 10^{-8}$ & 77.5 & 1.179 & 50.0 & 5.16 & 10.7 \\
\hline 12.59 & 79.4 & 1.250 & 51.9 & 5.28 & 10.34 \\
\hline 12.59 & 79.4 & 1.50 & 62.2 & 6.57 & 12.4 \\
\hline 8.93 & $1.12 \cdot 10^{7}$ & 2.59 & 76.3 & 5.92 & 5.39 \\
\hline 5.49 & 1.82 & 6.71 & $1.215 \cdot 10^{-4}$ & 6.12 & 2.02 \\
\hline 4.67 & 2.14 & 10.5 & 1.616 & 6.61 & 1.65 \\
\hline 3.16 & 3.16 & 23.3 & 2.43 & 7.36 & $0.77 \pm 0.05$ \\
\hline 2.34 & 4.27 & 57.8 & 4.46 & 10.20 & $0.58 \pm 0.14$ \\
\hline 1.48 & 6.76 & 200 & 9.77 & 14.31 & $0.32 \pm 0.15$ \\
\hline 1.41 & 7.07 & 227 & 10.56 & 14.75 & $0.30 \pm 0.10$ \\
\hline 1.41 & 7.07 & 118 & 5.48 & 7.59 & $0.16 \pm 0.06$ \\
\hline 1.41 & 7.07 & 140 & 6.51 & 9.04 & $0.18 \pm 0.07$ \\
\hline 1.29 & 7.75 & 417 & 17.75 & 22.77 & $0.38 \pm 0.12$ \\
\hline 1.27 & 7.85 & 625 & 26.20 & 33.1 & $0.54 \pm 0.40$ \\
\hline 0.79 & 12.6 & 1820 & 47.4 & 37.4 & $0.24 \pm 0.02$ \\
\hline
\end{tabular}




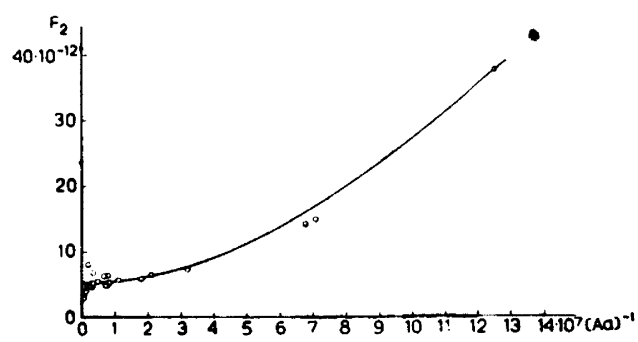

Fig. 7. $\mathrm{F}_{2}$ calculated according to Equation (310 as a function of $\left[\mathrm{Aa}^{-}\right]^{1^{-}}$. The intercept on the $F_{2}$-axis gives an approximate value of $\varphi_{2}$; the scattering of the points is to great to make any calculation of $\varphi_{3}$ possible from the slope of the line at $F_{2}=O$.

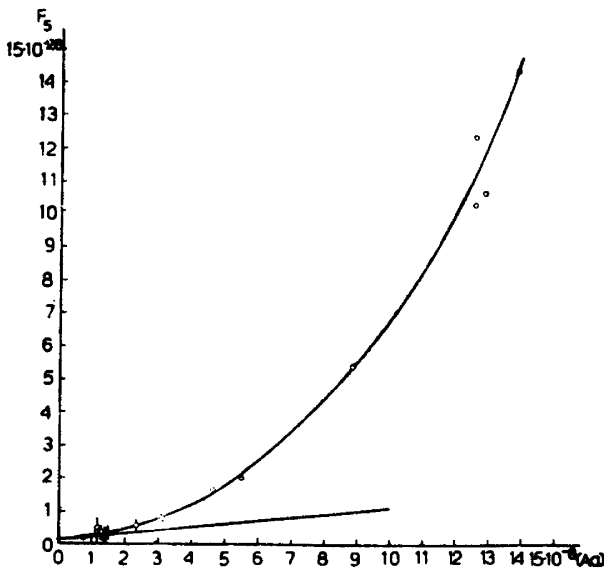

Fig. 8. $F_{5}$ calculated with Equation (32) as a function of $\left[\mathrm{Aa}^{-}\right]$. The intercept on the $F_{5}$-axis gives $\varphi_{4}$ and the slope of the line $\varphi_{3}$. The values of $F_{5}$ are given in Table 2 .

Table 3. Comparision of $\varphi_{n}$ obtained by method $A$ and $B$.

\begin{tabular}{|l|l|c|}
\hline & Method A & Method B \\
\hline & & \\
$\varphi_{1}$ & $1.13 \cdot 10^{-5}$ & $(1.0 \pm 0.1) 10^{-5}$ \\
$\varphi_{2}$ & $4.38 \cdot 10^{-12}$ & $(5.4 \pm 0.3) 10^{-12}$ \\
$\varphi_{3}$ & $1.63 \cdot 10^{-19}$ & $(1.0 \pm 0.2) 10^{-19}$ \\
$\varphi_{4}$ & $1.15 \cdot 10^{-27}$ & $(1.5 \pm 0.5) 10^{-27}$ \\
\hline
\end{tabular}

In Table 3 the values of $\varphi$ obtained by the two methods are compared. These values have been used in Equation (12b) to calculate $q$ as a function of [Aa]. The two resultant curves, $q_{\mathrm{A}}$ and $q_{\mathrm{B}}$, are shown in Fig. 9, where the symbols are taken from Table 1 . The difference between the two curves is very small; the closest agreement to the measured points is given by $q_{\mathrm{B}}$. With the values from Method B $k_{n}$ is calculated:

$$
\begin{aligned}
& k_{1}=(0.7 \pm 0.3) 10^{8} \\
& k_{2}=(5.4 \pm 1.7) 10^{7} \\
& k_{3}=(1.9 \pm 0.3) 10^{6} \\
& k_{4}=(1.0 \pm 0.1) 10^{5}
\end{aligned}
$$



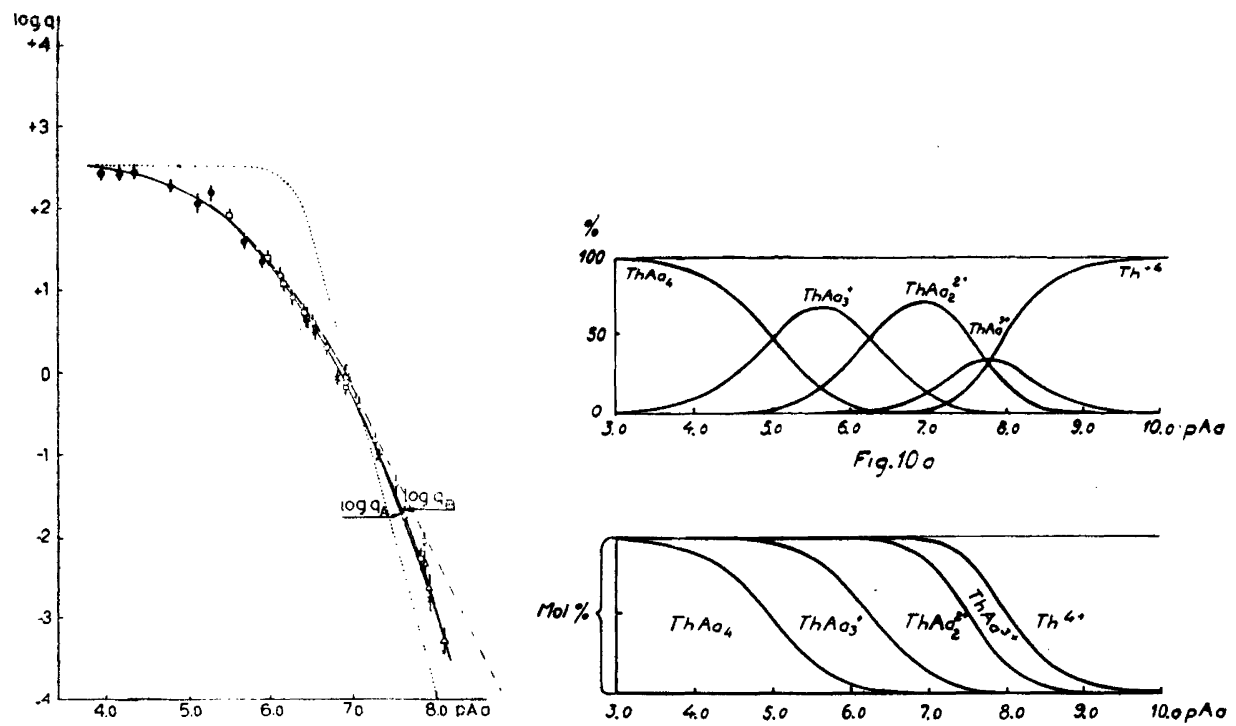

Fig. 9. $\log q$ is calculated as a function of $p A a$ under the following assumptions: The only thorium species existing in the aqueous phase are a/ $T h^{4+}$ and $T h A a_{4}$ (.....); b/ $T h A a_{4}, T h A a_{3}^{+}, T h A a_{2}^{2+}$ (- - - - ); cl all species $T h A a_{n}^{4-n}$ for $O \leq n \leq 4$ ( ). The experimental points are the same as in Figure 3.

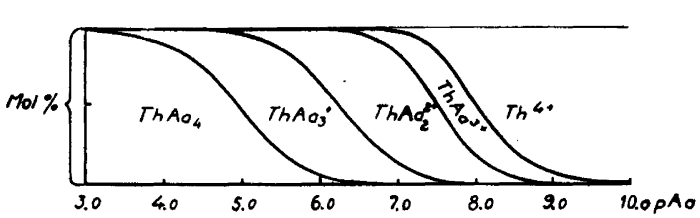

Fig. $10 \mathrm{a}$ and Fig. $10 \mathrm{~b}$. The percentage of the different complexes as a function of the concentration of acetylacetonate ions $(p A a=$ - $\log \left[\mathrm{Aa}^{-}\right]$) in two different graphical representations: in al the percentage of a complex for a certain $p A a$ is the height of the curve over the zero line; in b/ the percentage is the length of the vertical line at this $p A a$ which falls in the area of that complex.

Equation (22) is transformed to

$$
\alpha_{n}=\frac{\varphi_{4-n}[\mathrm{Aa}]^{n-4}}{\varphi_{1}[\mathrm{Aa}]^{-4}+\varphi_{3}[\mathrm{Aa}]^{-3}+\varphi_{2}[\mathrm{Aa}]^{-2}+\varphi_{1}[\mathrm{Aa}]^{-1}+1}
$$

$a_{n}$ is calculated with $\varphi$ from method B. Fig. 10a gives directly the percentage of the $n^{t h}$ complex $\left(=100 \alpha_{n}\right)$ at a certain pAa. The same result is given in an other graphical representation in Fig. $10 \mathrm{~b}$; the percentage of a complex is here given by the height of the vertical line which falls in the region of that complex for a certain pAa. Thus the areas in Fig. $10 \mathrm{~b}$ gives the ranges of existence of the individual complexes.

The true distribution factor of $\mathrm{ThAa}_{4}$ between benzene and water is given by Equation (9), where $\Lambda_{\mathrm{be}} / \Lambda_{\mathrm{aq}}=0.96$ and $\lambda^{\prime}=330 \pm 30$. We then have $\lambda_{4}=315 \pm 30$. This may be compared with the value of 80 for the distri- 
bution of $\mathrm{PuAa}_{4}$ between benzene and water, found by Harvey et al. ${ }^{19} \mathrm{How}$ ever they report no data on the concentration of acetylacetone.

\section{DISCUSSION}

In the calculations made here it has been presumed from the beginning that all complexes $\mathrm{ThAa}_{n}^{4-n}$ should exist. Attempts have been made to find if any complexes could be eliminated.

If we assume that the equilibrium between thorium and acetylacetone in water is completely described by

$$
\mathrm{Th}^{4+}+4 \mathrm{Aa}^{-} \rightleftharpoons \mathrm{ThAa}_{4}
$$

we can deduce the equation

$$
\left(\frac{\lambda_{4}^{\prime}}{q}-1\right)[\mathrm{Aa}]^{4}=\varkappa_{4}^{-3}
$$

Comparing Equations (32) and (34) it is seen that $F_{5}=\varkappa_{4}^{-1}$, if Equation (34) is valid. In Table 2 the values for $F_{5}$ are given; as no constant value is obtained, it may be concluded that the assumption for the deduction of Equation (34) is certainly wrong. The dotted line in Fig. 9 gives $q$ as a function of [Aa] according to Equation (34); $x_{4}^{-1}$ was chosen $3.3 \cdot 10^{-26}$. If the $x_{4}^{-1}$ value is varied the curve will be displaced along the pAa-axis retaining its form constant. It is easily seen that a good agreement with the measured $q$ cannot be obtained.

If we assume that $[\mathrm{Th}]=[\mathrm{ThAa}]=0$, we can obtain a curve, which fits the upper part of the experimental distribution curve very well; this gives the dashed line in Fig. 9. However the agreement with the experimental points is bad for $\log q<0$. Thus it may be concluded that all species $\mathbf{T h}^{4+}$, $\mathrm{ThAa}^{3+}, \mathrm{ThAa}_{2}^{2+}, \mathrm{ThAa}_{3}^{+}$and $\mathrm{ThAa}_{4}$ exist in the aqueous solution.

If negatively charged complexes like $\mathrm{ThAa}_{5}^{-}, \mathrm{ThAa}_{6}^{2-}$, etc., were formed, $q$ should reach a maximum at a certain $\mathrm{pAa}$, and then decrease for higher concentrations of $\mathrm{Aa}^{-}$. From Fig. 3 it is seen that $q$ approaches to a maximum. It has not been possible to make measurements on higher [Aa] than $\approx 10^{-4}$, and negative complexes may exist at still higher [Aa]. It is unlikely on steric grounds, however, that negative complexes are formed. The present results give no evidence of higher complexes than $\mathrm{ThAa}_{4}$. 


\section{SUMMARY}

The complexes of $\mathrm{Th}^{4+}$ and acetylacetone (HAa) in aqueous solution have been investigated by measuring the distribution of tracer amounts of $\mathrm{Th}^{234}$ $\left(\mathrm{UX}_{1}\right)$ between benzene and aqueous solutions of various $\left[\mathrm{Aa}^{-}\right]$. The measurements have been performed at $25^{\circ} \mathrm{C}$ and at an approximately constant ionic strength of $0.01 \mathrm{~mol} / 1$, which was brought about by adding $\mathrm{HClO}_{4}$, and $\mathrm{NaOH}$. The hydrolysis of $\mathrm{Th}^{4+}$ was found to be negligible, and no polynuclear complexes seemed to be formed at these low concentrations. The results could be explained only by assuming that all complexes $\mathrm{ThAa}^{3+}$, ThAa ${ }_{2}^{2+}$, $\mathrm{ThAa}_{3}^{+}$ and $\mathrm{ThAa}_{4}$ exist. The constants for the stepwise addition of $\mathrm{Aa}^{-}$to $\mathrm{Th}^{4+}$ $\left(k_{1}, k_{2}, \ldots\right)$ and those for the over all addition $\left(\bar{x}_{1}, x_{2}, \ldots\right)$ were calculated by two independent methods, and consistent results were obtained;

$$
\begin{array}{ll}
k_{1}=(0.7 \pm 0.3) 10^{8} & x_{1}=0.7 \cdot 10^{8} \\
k_{2}=(5.4 \pm 1.7) 10^{7} & x_{2}=3.8 \cdot 10^{15} \\
k_{3}=(1.9 \pm 0.3) 10^{6} & x_{3}=7.2 \cdot 10^{21} \\
k_{4}=(1.0 \pm 0.1) 10^{5} & x_{4}=7.2 \cdot 10^{26}
\end{array}
$$

The distribution constant of $\mathrm{ThAa}_{4}$ between benzene and water was found to be $\lambda_{4}=315 \pm 30$ !

I wish to thank the head of FOA 1, Professor Gustav Ljunggren, for helping me in many ways, and Professor Arne Ölander for the interest he has shown in this work. I am greatly indebted to Professor Lars Gunnar Sillén for the many valuable suggestions, which have proved so helpful to me. With my friends Laborator Roland Rynninger and Fil.mag. David Dyrssen I have had many fruitful discussions.

\section{REFERENCES}

1. Bjerrum, N. Z. anorg. Chem. 119 (1921) 189.

2. Sillén, L. G. Acta Chem. Scand. 3 (1949) 539.

3. Bjerrum, J. Metal ammine formation in aqueous solution. Diss. Copenhagen (1941).

4. Fronaeus, S. Diss. Lund (1948).

5. Calvin, M., and Wilson, K. W. J. Am. Chem. Soc. 67 (1945) 2003.

6. Hückel W. Anorganische Struckturchemie, Stuttgart (1948) p. 150.

7. Morse, H. Z. physik. Chem. 41 (1902) 709.

8. Grahame, D. C., and Seaborg, G. T. J. Am. Chem. Soc. 60 (1938) 2524.

9. Leden, I. Diss. Lund (1943), Z. physik. Chem. A 188 (1941) 160.

10. Ahrland, S. Acta Chem. Scand. 3 (1949) 374.

11. Biltz, W., and Clinch, J. A. Z. anorg. Chem. 40 (1904) 218.

12. Eidinoff, M. J. Am. Chem. Soc. 67 (1945) 2072.

13. Rydberg, J. Svensk Kem. Tid. 62 (1950) 179. 
14. Dyrssen, D. Svensk Kem. Tid. 62 (1950) 153.

15. Morgan, G. T., and Moss, H. W. J. Chem. Soc. 105 (1914) 189.

16. Grossman, P. Z. physik. Chem. 109 (1924) 349.

17. In Goodman, C. The science and engineering of nuclear power. $I$, Cambridge, Mass. (1947) p. 50.

18. Hietanen, S., and Sillén, L. G. Private communication.

19. Harvey, B. G., Heal, H. G., Maddock, A. G., and Rowley, E. L. J. Chem. Soc. (1947 II) 1010 .

20. Connick, R. E., and McVey, W. H. J. Am. Chem. Soc. 71 (1949) 3182.

21. Way, K., Fano, L., Scott, M. R., and Thew, K. Nuclear data, Washington (1950).

Received September 8, 1950. 